\title{
A New Joint Zero-Forcing Beamforming Algorithm for Massive MIMO System
}

\author{
Yingchao ZHOU ${ }^{1 *}$, Bin SHEN ${ }^{1 *}$, Qian WANG ${ }^{1}$, Dongmin $\mathrm{CHOI}^{2}$ \\ ${ }^{1}$ Chongqing University of Posts and Telecommunications, \\ Chongqing, 400065, China. \\ 394356457@qq.com, shenbin@cqupt.edu.cn (*Corresponding authors), \\ 1637965699@qq.com \\ ${ }^{2}$ Chosun University, \\ 309 Pilmun-daero, Dong-gu, Gwangju, South Korea. \\ jdmcc@chosun.ac.kr
}

\begin{abstract}
In massive multiple input multiple output (M-MIMO) systems where the number of antennas mounted at the base station (BS) is much larger than that of the mobile users, the existing beamforming schemes generally choose all users over the downlink as receivers. However, due to the fact that various channels may be significantly different, the existing solutions may not be appropriate in case that the number of users becomes sufficiently large, and hence the system throughput is not optimal. In addition, if all antennas at the BS are selected to transmit data streams, the hardware complexity requirement is consequently high, which results in the waste of RF chains and transmit power. In this paper, we propose a new zero-forcing beamforming algorithm on the basis of joint user grouping and antenna selection for M-MIMO systems. When a M-MIMO BS serves a number of users, we divide users into two groups and select an optimal antennas subset. The proposed user grouping method can maximize the total throughput by grouping users into two subsets and selecting the group with better channel station to receive data streams, and the antenna selection method aims to alleviate the system complexity and RF chains cost. The zero-forcing beamforming algorithm based on user grouping and antenna selection will greatly reduce the hardware complexity by lowering the cost and power consumption of radio-frequency chains with only a small performance loss. Simulations illustrate the proposed algorithm provides a better trade-off between the system throughput performance and the hardware complexity in M-MIMO systems.
\end{abstract}

Keywords: Massive MIMO, User grouping, Antenna selection, Zero-forcing beamforming, Hardware Complextity.

\section{Introduction}

The basic characteristics of M-MIMO technology lie in that the base station (BS) typically mounted with a large number of antennas serves a number of mobile users in the upcoming $5 \mathrm{G}$ mobile communication network. Compared to the number of antennas in $4 \mathrm{G}$, which is utmost four for the long term evolution (LTE) and up to eight for LTE Advanced (LTE-A), the number of antennas of M-MIMO system increases by one or two orders of magnitude [21, 23]. The users located within the coverage area of the BS use the degrees of freedom provided by large scale antennas array to communicate with BS simultaneously within the same time- frequency resource, which not only significantly increases the throughput $[3$, $12,15,6]$, and by orders of magnitude improves the spectrum efficiency, but also reduces the interference and enhances the robustness of the system [10, 11, 7, 19, 18]. Meanwhile, diversity gain and array gain it provides enable us to reduce the transmitted power and improve power efficiency significantly $[2,14]$.

In order to utilize the large number of antennas fully, one needs to implement as the same number of RF chains as that of the BS antennas, which gives rise to prohibitively high hardware complexity, impractically large power consumption, and infeasible fabrication cost. Fortunately, antenna selection is an efficient scheme by exploiting the spatial selectivity of the large size of M-MIMO antennas array to ameliorate the system gain in antenna diversity [16]. Practically, if the number of transmit antennas at the BS is large and the RF chains are subject to a limited number, the system performance can be enhanced significantly by means of finding a subset of the transmit antennas operating in salient channel environments. Various antenna selection algorithms proposed for conventional MIMO system can be found in $[13,17]$, but there are still few efforts dedicated to selecting the antennas to be activated for transmission in M-MIMO systems. To save the overhead used for acquiring the channel state information (CSI), the authors of [9] proposed a joint power and antenna selection optimization scheme for large scale distributed MIMO networks. Because the problem itself is intrinsically combinatorial and non-convex, it was decomposed into several subproblems that were separately solved to achieve the optimization. A random antenna selection scheme was proposed 
in [8], where the optimum number of antennas for a variety of circumstances was determined, and it was shown that the simple random antenna selection would yield a significant gain in energy efficiency. However, as for a perfect solution of random antenna selection, it is probably infeasible to implement it for M-MIMO systems. In [4], the original problem was solved with a semi-definite relaxation, where the selection parameter holding the values of either 0 or 1 was transformed from a discrete from into a continuous range of $[0,1]$ with a cutting-off threshold.

The above-mentioned issues are almost all about dealing with the antennas at the BS. As for the number of users that could be served simultaneously, it is practically limited by the number of transmit antennas. Additionally, the performance of M-MIMO systems relies on the user selection approaches essentially. The problem of user scheduling in MIMO systems has also been addressed widely in the literature. For example, the problem formulated for joint antenna selection and user scheduling was proposed to be solved in distributed M-MIMO systems under the constraint of backhaul capacity. In [20] a joint scheme of transmit antenna selection and user scheduling was proposed for maximizing the sum of data broadcasting rates and benefiting from both the gain of spatial selectivity and the gain of multiuser diversity brought about by antenna selection and user scheduling, respectively. However, user scheduling of the joint algorithm was performed over the semi-orthogonal user selection (SUS) algorithm, which demands in each iteration a channel matrix inversion and correspondingly requires a computation complexity of at most $O\left(N^{3}\right)$. The optimal strategy and solution for antenna selection and user scheduling may be perfectly achieved with an exhaustive search, e.g. brute force search (BFS), which is extremely for M-MIMO system in practice.

In this paper, we propose a new zero-forcing beamforming algorithm based on joint user grouping and antenna selection for M-MIMO system. Within the cell of interest, we divide all users into two groups: one group contains the target users to receive signals from the BS, and the other group is for users in idle mode, not operating to receive signal. With an aim to achieve a higher sum rate by selecting the users with better channel condition, on the other hand at the $\mathrm{BS}$, we process the transmit antennas by selecting an optimal antenna combination for each transmit data stream, in order that we can obtain the low complexity of hardware with acceptable performance, further lower RF circuit cost and save power consumption in M-MIMO systems.

Notations: Boldface lower and upper case symbols respectively stand for vectors and matrices. The transpose and Hermitian transpose operators are denoted by $(\cdot)^{T}$ and $(\cdot)^{H}$, respectively. The MoorePenrose pseudoinverse operator is represented by $(\cdot)^{-1}$. The 0 -norm and 2-norm of a vector is denoted by $\|\cdot\|_{0}$ and $\|\cdot\|$, respectively. The size of a set is denoted by $|\cdot|$.

\section{System Model}

We consider that a single cell equipped with $N$ transmit antennas at the BS is operating in a channel environment with quasi-static flat-fading effects and serving $M$ single antenna users $(N \geq$ $M)$. Let $\mathbf{H}=\left[\mathbf{h}_{1}^{H}, \mathbf{h}_{2}^{H}, \ldots, \mathbf{h}_{N}^{H}\right]^{H} \in \mathbb{C}^{M \times N}$ stand for the channel matrix of all the mobile users, where $\mathbf{h}_{k}=\left[h_{k 1}, h_{k 1}, \ldots, h_{k N}\right] \in \mathbb{C}^{1 \times N}$ is the user k's channel vector. Generally, the system is operating in TDD mode to obtain the perfect downlink CSI from the uplink channel sounding, relying on the channel reciprocity between them.

It is assumed that $\mathbb{U}_{\text {all }}=\{1,2, \cdots, M\}$ is the set of indices of all users in the cell, and $\mathbb{S}=\{\pi(1), \pi(2), \cdots, \pi(|\mathbb{S}|)\}$ is the set of indices of the optimal users selected to receive signals, for any $\mathbb{S} \subset \mathbb{U}_{\text {all }}$, where $\pi(i)$ is the $i$-th element of the optimal user index set $\mathbb{S}$, and the $\pi(i)$-th element of $\mathbb{U}_{\text {all }}$, corresponding to the $\pi(i)$-th user in the cell. Denote $|\mathbb{S}|$ as the size of set $\mathbb{S}$, that is the number of optimal users. The transmit signal vector $\mathbf{x}$ at the BS forms as a linear stream combination of all the selected users'data streams $\mathbf{S}$ with the zeroforcing beamforming (ZFBF) matrix $\mathbf{W}_{\mathbb{S}}$, by:

$\mathbf{x}=\mathbf{W}_{\mathbb{S}} \mathbf{P}_{\mathbb{S}}^{\frac{1}{2}} \mathbf{s}=\sum_{i=1}^{|\mathbb{S}|} \mathbf{w}_{\pi(i)} \sqrt{p_{\pi(i)}} s_{\pi(i)}$,

where $\mathbf{W}_{\mathbb{S}}=\left[\mathbf{w}_{\pi(1)}, \mathbf{w}_{\pi(2)}, \ldots, \mathbf{w}_{\pi(\mathbb{S})}\right] \in \mathbb{C}^{N \times \mathbb{S} \mid}$ is the ZFBF weight matrix for the optimal users, with $\mathbf{w}_{\pi(i)}=\left[w_{\pi(i) 1}, w_{\pi(i) 2}, \ldots, w_{\pi(i) N}\right]^{T} \in \mathbb{C}^{N \times 1} \quad, \quad p_{\pi(i)}$ is the $\pi(i)$-th transmit power scaling factor for $\mathbf{P}=\operatorname{diag}\left(p_{1}, p_{2}, \cdots, p_{\mathbb{S} \mid}\right)$ and $\mathbf{s}$ is the symbol vector of the BS transmitter. The received signal vector with respect to the optimal user set is: 


$$
\mathbf{y}=\mathbf{H}_{\mathbb{S}} \mathbf{x}+\mathbf{n}=\mathbf{H}_{\mathbb{S}} \mathbf{W}_{\mathbb{S}} \mathbf{P}_{\mathbb{S}}^{\frac{1}{2}} \mathbf{s}+\mathbf{n}
$$

w h e r e $\mathbf{H}_{\mathbb{S}}=\left[\mathbf{h}_{\pi(1)}^{H}, \mathbf{h}_{\pi(2)}^{H}, \cdots, \mathbf{h}_{\pi(\mid \mathbb{S})}^{H}\right]^{H} \in \mathbb{C}^{|\mathbb{S}| \times N}$ is the channel matrix of the optimal users, $\mathbf{h}_{\pi(i)}=\left[h_{\pi(i) 1}, h_{\pi(i) 2}, \cdots, h_{\pi(i) N}\right] \in \mathbb{C}^{1 \times N} \quad$ a $\quad n \quad d$ $\mathbf{n}=\left[n_{1}, n_{2}, \cdots, n_{|\mathbb{S}|)}\right]^{H} \in \mathbb{C}^{|\mathbb{S}| \times 1}$ is the white Gaussian noise vector with its entries being random variables with zero mean and unit variance, i.e. $C N(0,1)$. Specifically, the received signal of user $i$ is:

$$
y_{i}=\mathbf{h}_{\pi(i)} \mathbf{w}_{\pi(i)} \sqrt{p_{\pi(i)}} s_{\pi(i)}+\sum_{l=1, l \neq i}^{\mid \mathbb{S}} \mathbf{h}_{\pi(i)} \mathbf{w}_{\pi(l)} \sqrt{p_{\pi(l)}} s_{\pi(l)}+n_{\pi(i)},
$$

$$
\mathbf{W}_{\mathbb{U}}=\left[\mathbf{w}_{\pi(1)}, \mathbf{w}_{\pi(2)}, \cdots, \mathbf{w}_{\pi(\mathbb{U})}\right]=\mathbf{H}_{\mathbb{U}}^{H}\left(\mathbf{H}_{\mathbb{U}} \mathbf{H}_{\mathbb{U}}^{H}\right)^{-1},
$$

(4) is the equation of ZF beamforming matrix, where $\mathbb{U}=[\pi(1), \pi(2), \ldots, \pi(|\mathbb{U}|)]$ is the indices set of users to be selected, with $\mathbb{U} \subset \mathbb{U}_{\text {all }}$. The initial $\mathbb{U}$ represents all users, that means $\mathbb{U}=\mathbb{U}_{\text {all }}$. Similarly, the channel matrix and the beamforming matrix for $\mathbb{U}$ are $\mathbf{H}_{\mathbb{U}}=\left[\mathbf{h}_{\pi(1)}^{H}, \mathbf{h}_{\pi(2)}^{H}, \cdots, \mathbf{h}_{\pi(\mathbb{U})}^{H}\right]^{H} \in \mathbb{C}^{|\mathbb{U}| \times N}$ and $\mathbf{W}_{\mathbb{S}}=\left[\mathbf{w}_{\pi(1)}, \mathbf{w}_{\pi(2)}, \ldots, \mathbf{w}_{\pi(\mathbb{U})}\right] \in \mathbb{C}^{N \times|\mathbb{U}|}$. Assuming that $\mathbb{U} \backslash\{\mathrm{n}\}$ represents the new set that removes the element $n$ from the original set $\mathbb{U}, \mathbf{H}_{\mathbb{U} \backslash\{n\}}$ is the new channel matrix of all the selected users except user $n$ and $\mathbf{W}_{\mathbb{U} \backslash\{n\}}$ is the column-reduced beamforming matrix. The sum rate of the user set $\mathbb{U}$ is:

$$
\begin{aligned}
R(\mathbb{U})= & \log _{2}\left[\operatorname{det}\left(\mathbf{I}_{\mathbb{U}}+\mathbf{H}_{\mathbb{U}} \mathbf{W}_{\mathbb{U}} \mathbf{P}_{\mathbb{U}} \mathbf{W}_{\mathbb{U}}^{H} \mathbf{H}_{\mathbb{U}}^{H}\right)\right] \\
= & \sum_{p_{i}: \sum_{\pi(i) \in \mathbb{U}}^{\pi(i) \in \mathbb{U},}, \lambda_{i}^{-1} p_{i} \leq P} \log _{2}\left(1+p_{i}\right),
\end{aligned}
$$

where

$$
\lambda_{i}=\frac{1}{\| \mathbf{w}_{i}^{2}},
$$

is the effective channel coefficient of user $\pi(i)$ [22], and hence $\lambda_{i}^{-1} p_{i}$ is the transmit power of user $\pi(i)$ with $P$ denoting the total transmit power. $\mathbf{I}_{\mathbb{U}}$ is the $|\mathbb{U}| \times|\mathbb{U}|$ identity matrix. The optimal power scaling factor $p_{i}$ can be found by waterfilling. First, we construct the Lagrangian function:

$$
L=\sum_{\pi(i) \in \mathbb{U}} \log _{2}\left(1+p_{i}\right)+\mu\left(\sum_{\pi(i) \in \mathbb{U}} \lambda_{i}^{-1} p_{i}-P\right),
$$

indent then taking a derivative of $L$ with respect to $p_{i}$, we obtain $\frac{\partial L}{\partial p_{i}}$ as: $\frac{\partial L}{\partial p_{i}}=\frac{1}{1+p_{i}} \frac{1}{\ln 2}+\mu \lambda_{i}^{-1}=0$

solving the equation above, we find the optimal transmit power for user $\pi(i)$ is:

$p_{i}=\left(\mu \lambda_{i}-1\right)^{+}$

where $(x)^{+}=\max \{x, 0\}$, and $\mu$ is the water level that satisfies:

$\sum_{i \in \mathbb{U}}\left(\mu-\lambda_{i}^{-1}\right)^{+}=P$.

\section{Joint Beamforming Algorithm}

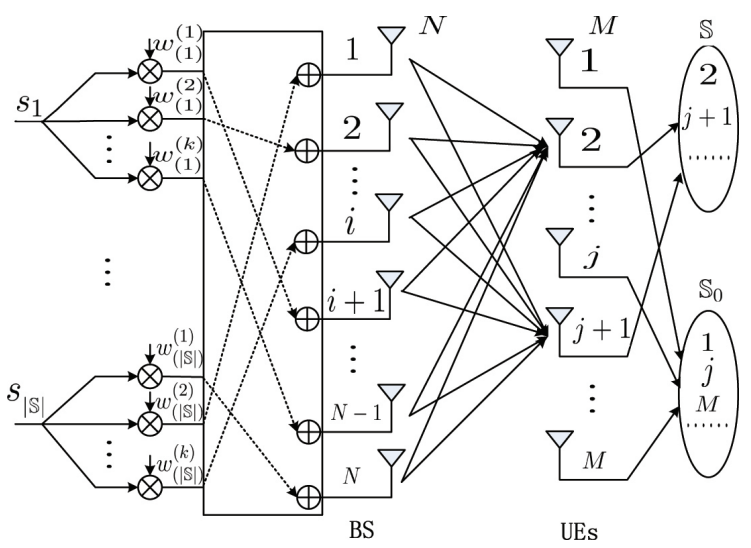

Figure 1. Illustration of joint user grouping and antenna selection ZF beamforming algorithm

Joint user grouping and antenna selection beamforming algorithm is investigated and proposed in this paper for M-MIMO transmission systems, as shown in Figure 1. In the proposed scheme, we consider combining schemes of user grouping within a cell and antenna selection at the BS, which means that at the side of the cell all users are divided into two groups: one group is selected to receive signal, namely the target user set $\mathbb{S}$, and the other group is not operating in receive mode, denoted as $\mathbb{S}_{0}$; at the same time, selecting an optimal antenna subset for the limited RF chain to alleviate the hardware complexity. The design problem is formulated as follow:

$$
\begin{array}{rc}
\text { (P1) maximize } & R(\mathbb{U}) \\
\text { subject to } & \mathbb{U} \subset \mathbb{U}_{\text {all }} \\
\| \mathbf{w}_{\pi(i)} 0 & =K, \pi(i) \in \mathbb{U} \\
& \sum_{i=1}^{|\mathbb{U}|} \| \mathbf{w}_{\pi(i)}{ }^{2} p_{i} \leq P,
\end{array}
$$

where (P1-II), (P1-III), (P1-IV) are respectively the constraint of user grouping and antenna 
selection, and the power constraint of the method. The 0-norm in (P1-III) represents that in the set each column of the beamforming matrix has $K$ nonzero elements and all the rest elements are zero, that means the number of transmit antennas selected at BS.

\subsection{User Grouping at the Receiver Side}

The proposed scheme at the receiver side uses a decremental user grouping scheme, which deletes the user with the minimum effective channel gain of the beamforming matrix per iteration. The algorithm first begins to select all users as the target users to receive signal, then deletes the user with the minimum effective channel gain $\lambda_{n}$ per iteration until the sum rate increment $\Delta R=R(\mathbb{U} \backslash\{n\})-R(\mathbb{U})<0$, and subsequently finds the set $\mathbb{S}$ of indices of optimal users and the ZF beamforming matrix $\mathbf{W}_{\mathbb{S}}$. The power allocation matrix $\mathbf{P}_{\mathbb{S}}$ is given by waterfilling in previous section.

Without the constraints (P1-III) and (P1-IV) in user grouping, we only need to consider:

$$
\begin{array}{ll}
\text { maximize } & R(\mathbb{U}) \\
\text { subject to } & \mathbb{U} \subset \mathbb{U}_{\text {all }}
\end{array}
$$

ZFBF algorithm carries out the operations of channel matrix inversion at the BS for the purpose of creating orthogonal downlink channels between the BS and the users without requiring user cooperation. The set of users' indices to be selected will change because of deleting the user with effectively minimum channel gain. Denoting the updated set of indices of the users $\mathbb{U}$ as $\widetilde{\mathbb{U}}$, and $\widetilde{U}=\mathbb{U} \backslash\{n\}$, we can obtain the beamforming vector $\mathbf{w}_{i}$ through the effective channel vector $(\mathrm{ECV}) \mathbf{v}_{i}$ defined by [5]:

$\mathbf{v}_{i}=\mathbf{h}_{i} \mathbf{P}_{i}^{\perp}$,

$\mathbf{w}_{i}=\frac{\mathbf{v}_{i}^{H}}{\| \mathbf{v}_{i}^{2}}$,

wh ere $\mathbf{P}_{i}^{\perp}=\mathbf{I}_{N}-\mathbf{H}_{\mathbb{U} \backslash\{i\}}^{H}\left(\mathbf{H}_{\mathbb{U} \backslash\{i\}} \mathbf{H}_{\mathbb{U} \backslash\{i\}}^{H}\right)^{-1} \mathbf{H}_{\mathbb{U} \backslash\{i\}}$ is the orthogonal projector matrix on the subspace $\mathbf{V}_{n}=\operatorname{span}\left\{\mathbf{h}_{j} \mid j \in \mathbb{U}, j \neq n\right\}$. The effective channel gain of user $i$ is $\lambda_{\mathrm{i}}=\left\|\mathrm{v}_{\mathrm{i}}\right\|^{2}$. The updated effective channel vector is given by:

$$
\begin{aligned}
& \tilde{\mathbf{v}}_{i}=\mathbf{h}_{i}\left(\mathbf{I}_{N}-\mathbf{H}_{\mathrm{v} \backslash\{i\}}^{H}\left(\mathbf{H}_{\mathrm{t} \backslash\{i\}} \mathbf{H}_{\mathrm{t} \backslash\{i\}}^{H}\right)^{-1} \mathbf{H}_{\mathrm{t} \backslash\{i\}}\right) \\
& =\mathbf{h}_{i}\left[\begin{array}{ll}
\mathbf{v}_{i}^{H} & \mathbf{v}_{n}^{H}
\end{array}\right]\left[\begin{array}{cc}
\mathbf{v}_{i} \mathbf{v}_{i}^{H} & \mathbf{v}_{i} \mathbf{v}_{n}^{H} \\
\mathbf{v}_{n} \mathbf{v}_{i}^{H} & \mathbf{v}_{n} \mathbf{v}_{n}^{H}
\end{array}\right]^{-1}\left[\begin{array}{c}
\mathbf{v}_{i} \\
\mathbf{v}_{n}
\end{array}\right] \\
& =\left[\begin{array}{ll}
\mathbf{h}_{i} \mathbf{v}_{i}^{H} & 0
\end{array}\right] \frac{1}{\left\|\mathbf{v}_{i}\right\|^{2}\left\|\mathbf{v}_{n}\right\|^{2}-\left\|\mathbf{v}_{i} \mathbf{v}_{n}^{H}\right\|^{2}} \\
& \cdot\left[\begin{array}{cc}
\mathbf{v}_{n} \mathbf{v}_{n}^{H} & -\mathbf{v}_{i} \mathbf{v}_{n}^{H} \\
-\mathbf{v}_{n} \mathbf{v}_{i}^{H} & \mathbf{v}_{i} \mathbf{v}_{i}^{H}
\end{array}\right]\left[\begin{array}{c}
\mathbf{v}_{i} \\
\mathbf{v}_{n}
\end{array}\right] \\
& =\frac{\left\|\mathbf{v}_{i}\right\|^{2}\left\|\mathbf{v}_{n}\right\|^{2}}{\left\|\mathbf{v}_{i}\right\|^{2}\left\|\mathbf{v}_{n}\right\|^{2}-\left\|\mathbf{v}_{i} \mathbf{v}_{n}^{H}\right\|^{2}}\left(\mathbf{v}_{i}-\frac{\mathbf{v}_{i} \mathbf{v}_{n}^{H}}{\left\|\mathbf{v}_{n}\right\|^{2}} \mathbf{v}_{n}\right)
\end{aligned}
$$

The updated effective channel gain and effective channel vector are respectively:

$$
\begin{aligned}
\tilde{\lambda}_{i} & =\left\|\tilde{\mathbf{v}}_{i}\right\|^{2}=\lambda_{i} \frac{\left\|\mathbf{v}_{i}\right\|^{2}\left\|\mathbf{v}_{n}\right\|^{2}}{\left\|\mathbf{v}_{i}\right\|^{2}\left\|\mathbf{v}_{n}\right\|^{2}-\left\|\mathbf{v}_{i} \mathbf{v}_{n}^{H}\right\|^{2}} \\
& =\frac{\lambda_{i}^{2} \lambda_{n}}{\lambda_{i} \lambda_{n}-\left\|\mathbf{v}_{i} \mathbf{v}_{n}^{H}\right\|^{2}}, \\
\tilde{\mathbf{V}}_{i} & =\frac{\lambda_{i} \lambda_{n}}{\lambda_{i} \lambda_{n}-\left\|\mathbf{v}_{i} \mathbf{v}_{n}^{H}\right\|^{2}}\left(\mathbf{v}_{i}-\frac{\mathbf{v}_{i} \mathbf{v}_{n}^{H}}{\lambda_{n}} \mathbf{v}_{n}\right)
\end{aligned}
$$

By plugging $\mathbf{w}_{i}=\frac{\mathbf{v}_{i}^{H}}{\| \mathbf{v}_{i}{ }^{2}}$ and $\lambda_{\mathrm{i}}=\left\|\mathrm{v}_{\mathrm{i}}\right\|^{2}$ into (14) and (15), we obtain the updated $\tilde{\lambda}_{i}$ on the basis of the beamforming vector $\mathbf{w}_{i}$ as:

$$
\begin{gathered}
\tilde{\lambda}_{i}=\frac{\lambda_{i}}{1-\lambda_{n} \lambda_{i}\left|\mathbf{w}_{n}^{H} \mathbf{w}_{i}\right|^{2}} \\
\tilde{\mathbf{W}}_{i}=\mathbf{w}_{i}-\lambda_{n} \mathbf{w}_{n}^{H} \mathbf{w}_{i} \mathbf{w}_{n} .
\end{gathered}
$$

Let $\mathbb{U}$ denote the index set of users to be selected and the user grouping algorithm is generally carried out with the following steps. Step 1) Initialization:

$$
\begin{aligned}
& \mathbb{U}=\{1,2, \cdots, M\} \\
& \lambda_{i}=\frac{1}{\| \mathbf{w}_{i}{ }^{2}}, \quad i \in \mathbb{U}
\end{aligned}
$$

where $\left[\mathbf{w}_{1}, \mathbf{w}_{2}, \cdots, \mathbf{w}_{M}\right]=\mathbf{H}^{H}\left(\mathbf{H H}^{H}\right)^{-1}$.

Step 2) Remove the user with the effectively minimum gain $\lambda_{n}$.

$n=\arg \min _{i \in \mathbb{U}}\left\{\lambda_{i}\right\}$, 
If $\Delta R=R(\mathbb{U} \backslash n)-R(\mathbb{U}) \geq 0$, we delete the user $n$, update $\mathbb{U}, \mathbf{w}_{i}, \lambda_{i}$ by formula (16), (17), and then repeat step 2).

$$
\begin{aligned}
& \mathbb{U} \leftarrow \mathbb{U} \backslash\{n\} \\
& \mathbf{w}_{i} \leftarrow \tilde{\mathbf{w}}_{i}=\mathbf{w}_{i}-\lambda_{n} \mathbf{w}_{n}^{H} \mathbf{w}_{i} \mathbf{w}_{n}, \quad i \in \mathbb{U} \\
& \lambda_{i} \leftarrow \tilde{\lambda}_{i}=\frac{\lambda_{i}}{1-\lambda_{n} \lambda_{i}\left|\mathbf{w}_{n}^{H} \mathbf{w}_{i}\right|^{2}}, \quad i \in \mathbb{U}
\end{aligned}
$$

Otherwise, go to step 3 ). Step 3) Exit from the iteration, the optimal users group $\mathbb{S}$ and the corresponding beamforming matrix are calculated as:

$$
\begin{aligned}
& \mathbb{S}=\{\pi(1), \pi(2), \cdots, \pi(|\mathbb{S}|)\}, \\
& \mathbf{W}_{\mathbb{S}}=\left[\mathbf{w}_{\pi(1)}, \mathbf{w}_{\pi(2)}, \cdots, \mathbf{w}_{\pi(\mathbb{S})}\right],
\end{aligned}
$$

To sum up, step 1) initializes all users, that is $\mathbb{U}=\mathbb{U}_{\text {all }}$, and computes the beamforming vector $\mathbf{w}_{i}$ and the effective channel gain $\lambda_{i}$ according to (4) and (6), respectively. Step 2) is an iteration process and the number of iterations only needs to be less than or equal to $M$. In this iterative process, the user $n \in \mathbb{U}$ with the effectively minimum channel gain is removed from $\mathbb{U}$ if the deletion of user $n$ does not decrease the sum rate, and then each parameter is updated. When entering step 3), the final users set and the optimal beamforming matrix can be calculated.

\subsection{Antenna Selection at BS}

Based on the proposed user grouping algorithm, the strategy of antenna selection is considered in this subsection for designing the joint scheme. A tremendously critical factor that increases the number of antennas in practical M-MIMO system is the cost of the RF chain comprised of analog to digital converters (ADCs), mixers, and low noise amplifiers. The hardware complexity we aim to reduce mainly lies in the number of RF chains in this paper. In the proposed scheme of beamforming, each stream for transmission is firstly multiplied with $K$ complex gains and then allocated to $K$ out of the $N$ available transmit antennas. Subsequently, signals that are assigned to the same transmit antenna are superimposed and transmitted over the antenna assigned to it. It means each of the channel beamforming column vectors in the beamforming matrix has $K$ nonzero entries and all the rest elements are exactly zero. The main difficulty in beamforming lies in searching for $K$ best antennas for each of the transmit data streams.

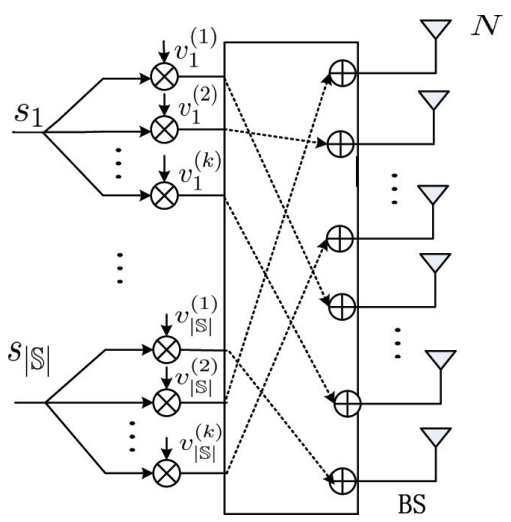

Figure 2. The antenna selection architecture at BS for M-MIMO

Figure 2 shows the antenna selection architecture at BS. The BS is supposed to have $N$ transmit antennas and on the receiver side there is virtually viewed to be $|\mathbb{U}|$ receive antennas. When we have $K=1$, the proposed beamforming scheme is exactly equivalent to the conventional point-topoint transmit antenna selection method with the lowest hardware complexity and the worst rate performance (Degrees of freedom are actually lost in this case). When it is for the case $K=N$, the proposed scheme will boil down to the general zero-forcing beamforming with the optimal rate performance and the highest complexity by selecting all antennas as transmitters. However, when $1<K<N$, this scheme provides a tradeoff between the hardware complexity and the rate performance by selecting some from all antennas to transmit signal.

The proposed antenna selection algorithm works as follows: selecting $k$ antennas at BS as the transmit antennas, that is to say, each of the channel beamforming column vectors has $K$ non-zero entries and all of the rest elements are exactly zero.

The best way of acquiring the optimal $K$ would be the exhaustive search method, i.e., selecting a best one from all the possible antenna combinations to maximize the throughput. However, because the complexity order of this optimal method is $O\left(\left(C_{N}^{K}\right)^{M}\right)$, which requires $C_{N}^{K}$ operations for each data stream, the larger the number of antennas, the higher the complexity, which undoubtedly results in that the actual system cannot realize the real-time data processing. The algorithm that we propose in this paper just utilizes the iterative shrinkage thresholding 
algorithm (ISTA) in [1]. Compared with the exhaustive search method, the algorithm reduces the computational complexity with the feasible realization of antenna selection.

The design problem of $K$-regular beamforming is formulated as:

(P2) maximise

$$
\begin{gathered}
R(\mathbb{U}) \\
\left\|\mathbf{w}_{\pi(i)}\right\|_{0}=K, \pi(i) \in \mathbb{U} \\
\sum_{i=1}^{\mathbb{U} \mid}\left\|\mathbf{w}_{\pi(i)}\right\|^{2} p_{i} \leq P,
\end{gathered}
$$$$
\text { subject to }
$$

where

$R(\mathbb{U})=\log _{2}\left[\operatorname{det}\left(\mathbf{I}_{|\mathbb{U}|}+\mathbf{H}_{\mathbb{U}} \mathbf{W}_{\mathbb{U}} \mathbf{P}_{\mathbb{U}} \mathbf{W}_{\mathbb{U}}^{H} \mathbf{H}_{\mathbb{U}}^{H}\right)\right], \quad \mathbf{H}_{\mathbb{U}}$ and $\mathbf{W}_{\mathbb{U}}$ are respectively the channel matrix and the beamforming matrix for the set of the users after deleting a users during the user grouping iterations. $\mathbf{P}_{\mathbb{U}}$ is the transmit power scaling matrix.

Therefore, we propose in this subsection an iterative algorithm for (P2) on the basis of zeroforcing beamforming that results in better system performance with reduced system complexity. It is worth noting that the problem (P2-I, P2-II), without (P2-III), is equivalently an optimization problem under the $l_{0}$-norm constraints. In this case, we need to minimize the sum rate cost in (P2-I) under the $l_{0}$-norm regularity constraints (P2-II) and the power constraints (P2-III). In order to circumvent the barriers in finding the solution, we substitute the $l_{0}$-norm constraints for the $K$-regularity with $l_{1}$-norm constraints by using compressed sensing to turn the non-convex constraints into a convex one. Then we adopt the ISTA algorithm to solve the complicated problem. Correspondingly, the relaxed problem for (P2) is given as follows:

(P3) minimize

$$
f(\overline{\mathbf{W}})
$$

subject to

$$
\begin{aligned}
& \left\|\overline{\mathbf{w}}_{i}\right\|_{1} \leq \xi, i \in \mathbb{U} \\
& \sum_{i=1}^{|\mathbb{U}|}\left\|\overline{\mathbf{w}}_{i}\right\|^{2} \bar{p}_{i} \leq P,
\end{aligned}
$$

where $\xi \geq 0$, and $\mathbb{U}$ is the optimal user set after users grouping operation. The relaxed problem need to satisfy that:

$$
\begin{aligned}
& \overline{\mathbf{w}}_{i}=\frac{\hat{\mathbf{w}}_{i}}{\| \hat{\mathbf{w}}_{i}}, i \in \mathbb{U}, \\
& \overline{\mathbf{P}}=\hat{\mathbf{P}} \cdot \operatorname{diag}\left(\left\|\hat{\mathbf{w}}_{1}\right\|^{2},\left\|\hat{\mathbf{w}}_{2}\right\|^{2}, \ldots\left\|\hat{\mathbf{w}}_{|\mathbb{U}|}\right\|^{2},\right), \\
& f(\overline{\mathbf{W}}) \triangleq-\log _{2}\left[\operatorname{det}\left(\mathbf{I}+\overline{\mathbf{H}} \overline{\mathbf{W}} \mathbf{P} \overline{\mathbf{W}}^{H} \overline{\mathbf{H}}^{H}\right)\right] .
\end{aligned}
$$

where $\overline{\mathbf{H}}, \mathbf{W}_{\mathbb{U}}, \mathbf{P}_{\mathbb{U}}$ are respectively the channel matrix, zero-frocing beamforming matrix and the optimal power scaling factor of the optimal users. In a general manner, a convex optimization problem can be expressed under the $l_{1}$-norm constraint as:

minimize $f(\mathbf{x})$

subject to $\|\mathbf{x}\|_{1} \leq c$

Constructing a Lagrangian function for the convex optimization with $\lambda \geq 0$, we have:

$\min f(\mathbf{x}+\|\mathbf{x}\|, \quad x \in$

Adopting the fundamental gradient idea to solve the $l_{1}$ regularized problem, we find out that since the $l_{1}$-norm is separable, the computation of $\mathbf{x}_{k}$ is reduced as the one solving a problem of onedimensional minimization for each component of itself, where by simple calculation it produces:

$\mathbf{x}_{k}=\mathcal{T}_{\lambda t_{k}}\left(\mathbf{x}_{k-1}-t_{k} \nabla f\left(\mathbf{x}_{k-1}\right)\right)$,

where $\mathcal{T}_{\lambda t_{k}}$ is the shrinkage operator defined by:

$\mathcal{T}_{\alpha}(x)_{i}=\left(\left|x_{i}\right|-\alpha\right)_{+} \operatorname{sign}\left(x_{i}\right)$,

where

$\mathcal{T}_{\alpha}(x)_{i}=\left\{\begin{array}{l}\left.\left|x_{i}\right|-\alpha\right)_{+} \operatorname{sign}\left(x_{i}\right), \text { if } x_{i} \text { is a real value } \\ \left(\left|x_{i}\right|-\alpha\right)_{+} e^{j \theta}, \quad \text { otherwise }\end{array}\right.$

and

$\left|x_{i}\right|-\alpha= \begin{cases}\left|x_{i}\right|-\alpha, & \text { if }\left|x_{i}\right| \geq \alpha \\ 0, & \text { otherwise }\end{cases}$

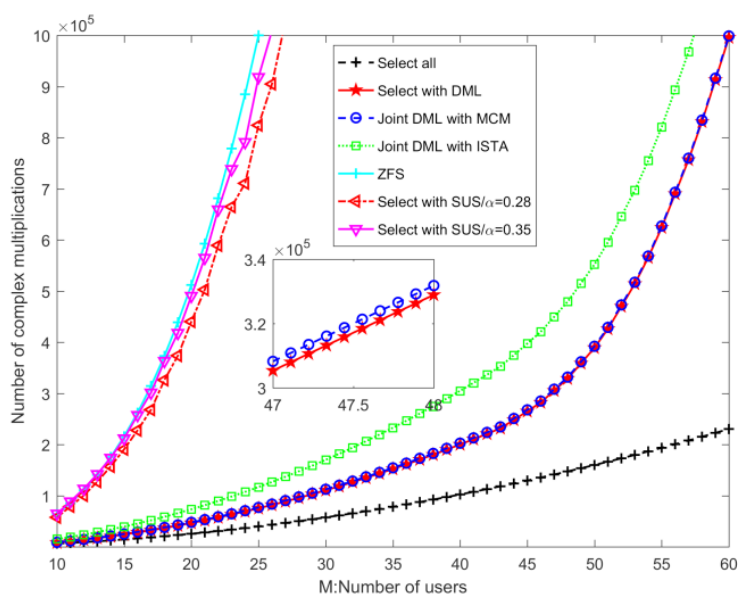

Figure 3. The computational complexity about number of complex multiplications of 'Select all', DML, ZFS, SUS (with different $\alpha$ ), and the joint beamforming algorithm about DML with MCM and the proposed joint DML with ISTA with $N=64$, $M=10 \sim 64$ and $S N R=20 d B$. 
Note that the problem (P3) without the power constraints can be formulated as an $l_{1}$-regularized optimization problem and the ISTA can be employed to solving the problem. However, we actually have the power constraints (P3-III) in practice. Aiming to incorporate the power constraints (P3-III), we need to normalize the power by:

$\mathcal{L}\left(\overline{\mathbf{w}}_{i}\right)=\left\{\begin{array}{l}\overline{\mathbf{w}}_{i} /\left\|\overline{\mathbf{w}}_{i}\right\|_{2}, \quad \text { if }\left\|\overline{\mathbf{w}}_{i}\right\|_{2}>1 \\ \overline{\mathbf{w}}_{i}, \quad \text { othertwise }\end{array}\right.$,

when $\overline{\mathbf{W}}=\left[\overline{\mathbf{w}}_{1}, \overline{\mathbf{w}}_{2}, \cdots, \overline{\mathbf{w}}_{\mid \mathbb{U}]}\right] \in \mathcal{C}^{N \times|\mathbb{U}|}$, the operation is just an extension of the classical gradient descent algorithm by means of proximal regularization for the column vector. Therefore, the beamforming matrix $\mathbf{W}_{\mathbb{U}}$ at each update is given by:

$$
\begin{aligned}
& \overline{\mathbf{W}}=\mathcal{L}^{(\mid \mathbb{U}) \circ} \mathcal{L}^{(|\mathbb{U}|-1) \circ} \ldots \mathcal{L}^{(1)} \bullet \\
& \mathcal{T}_{\lambda t_{k}}^{(\mathbb{U} \mid) \circ} \mathcal{T}_{\lambda t_{k}}^{(\mathbb{U} \mid-1) \circ} \ldots \mathcal{T}_{\lambda t_{k}}^{(1)}(\overline{\mathbf{W}}-\mu \nabla f(\overline{\mathbf{W}})
\end{aligned}
$$

where $\mathcal{L}^{(i)}$ is the normalization of $i$-th column of $\overline{\mathbf{W}}, \mathcal{T}_{\lambda t_{i}}^{(i)}$ is the shrinkage operator for the $i$-th column of $\overline{\mathbf{W}}$, and the gradient of $f(\overline{\mathbf{W}})$ is:

$$
\nabla f(\overline{\mathbf{W}})=-\overline{\mathbf{H}}^{H}\left(\mathbf{I}+\overline{\mathbf{H}} \mathbf{W} \overline{\mathbf{P}} \overline{\mathbf{W}}^{H} \overline{\mathbf{H}}^{H}\right)^{-1} \overline{\mathbf{H}} \overline{\mathbf{W}} \overline{\mathbf{P}}
$$

Note that in (35), $\circ$ and - respectively denotes the normalized and the shrinkage operator for every column.

In this paper, we relax the original $l_{0}$-norm regularity constraints to the $l_{1}$-norm constraints and solved (P3), which results in a larger number of the final output being non-zero elements than $k$. In addition, we need a next step to acquire a final solution satisfying the constraints given in (P1). In order to obtain the optimal non-zero elements, we select $K$ elements with the largest absolute values for each column of $\overline{\mathbf{W}}$ obtained from the ISTA algorithm. This column vector with $k$ nonzero elements needs to be normalized to satisfy the power constraints.

\subsection{Complexity Analysis}

The complexity of joint user grouping and antenna selection beamforming algorithm mainly comes from the initialization step of the Moore-Penrose pseudo-inverse of $\mathbf{W}$, involving a complexity of $O\left(N M^{2}\right)$, and the computation of the gradient (36), which is on the order of $O\left(N|\mathbb{S}|^{2}\right)$. In the sequel, the computations involved in the remaining steps of the proposed algorithm are analyzed and we can show the additional complexity is not larger than $O\left(N M^{2}\right)$.

The corresponding initialization of $\lambda_{i}$ in step 1) involves $M$ 2-norms operation of $1 \times N$ vectors, which introduces complex multiplications. The updating operation of $\mathbf{w}_{i}$ and $\lambda_{i}$ in step 2) includes $|\mathbb{S}|-1$ vector-vector multiplications and $|\mathbb{S}|-12$-norms operation, which involves $2 N(|\mathbb{S}|-1)$ complex multiplications. Hence, the total computational complexity of user grouping is:

$M N+\sum_{n=|\mathbb{S}|}^{M} 2 N(n-1) \ll M N+\sum_{n=1}^{M} 2 N(n-1)=N M^{2}$

While the computational complexity of antenna selection lies in the finding of the first $k$ nonzero elements with largest absolute values, it involves a complexity of $O(N|\mathbb{S}|)$. The total complexity of the proposed beamforming scheme based on joint user grouping and antenna selection is:

$O\left(N M^{2}+N|\mathbb{S}|^{2}+M N+\sum_{n=|\mathbb{S}|}^{M} 2 N(n-1)+N|\mathbb{S}|\right)$.

We have shown that the complexity of "Select All" method is $O\left(N M^{2}\right)$, because of the MoorePenrose seudo-inversion of $\mathbf{H}_{\mathbb{U}_{\text {all }}}$, which is lower yet with poor performance.

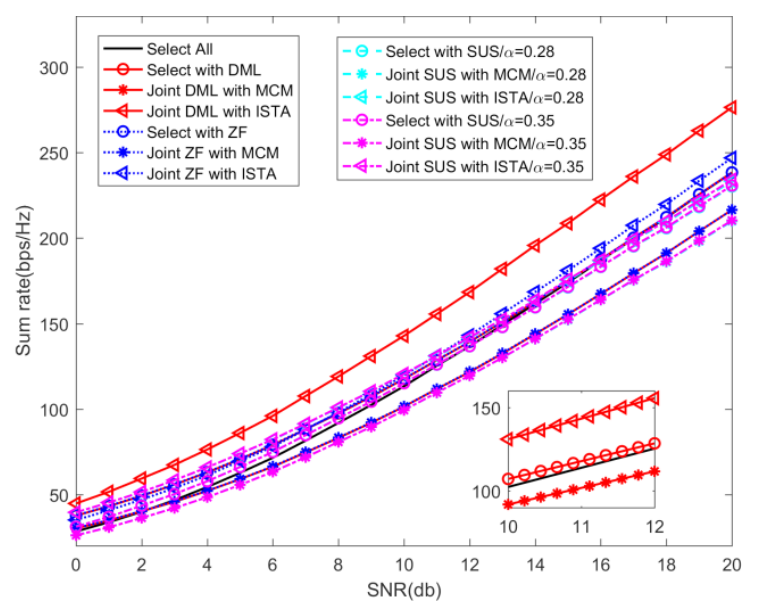

Figure 4. Sum rate performance comparison of different users grouping and antennas selection approach with $N=64, M=40, K=40$ and $S N R=0 \sim 20 \mathrm{~dB}$. 
From Figure 5, we can see the computation complexity of the proposed scheme is basically the same as that of the "Select All" method. The improvement in saving the computation complexity is significant over the previously mentioned user selection algorithms, such as ZFS and SUS, which are both assessed to have the complexity of $O\left(N M^{3}\right)$. Especially, it is noticed that the complexity of SUS scheme is highly sensitive to the choice of threshold $\alpha$.

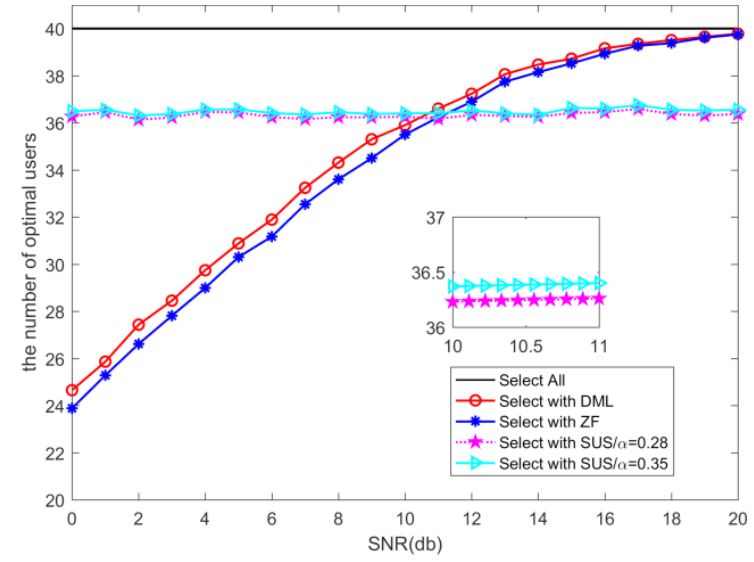

Figure 5. The comparison of the number of optimal user grouping under 'Select All', DML, ZFS, SUS (with different $\alpha$ ), $N=64, M=40, K=40$ and $S N R=$ $0 \sim 20 \mathrm{~dB}$.

\section{Simulations and Analysis}

In this section, computer simulations are carried out to evaluate the performance of DML, ZFS, SUS (with $\alpha=0.28$ and $\alpha=0.35$, respectively), 'Select All', and the proposed joint beamforming scheme with different antenna selection strategies, namely MCM and ISTA based on ZFBF. The BS is assumed to be equipped with $N=64$ antennas with the transmit SNR as $20 d B$. All curves are obtained by averaging over $10^{3}$ independent channel coefficient matrices where each entry is generated from a circular symmetric complex Gaussian random variable with zero mean and unit variance.

The sum rate and the number of selected users within a SNR range from $0 d B$ to $20 d B$ in Figure 4 and Figure 5 are compared. From Figure 4, we can see that the sum rate of the proposed joint DML and ISTA approch is highest than others. From the same coloured lines, which are grouped with the same method, it is clear to see antenna selection with ISTA is better than that with MCM, which is also illustrated in Figure 9. As shown in Figure 5, the optimal number of selected users increases with SNR, and the number of the method with DML is slightly higher than that with $\mathrm{ZF}$, but according to Figure 3, it reduces the computational complexity.

Figure 6 and Figure 7 show the sum rate and the number of optimal users with different number of users $M$. In Figure 6, the proposed algorithm based on joint DML and ISTA achieve a very good performance with a small $K$, that is $K=10$, and a optimal users group that the number is smaller than initialization as shown in Figure 6.

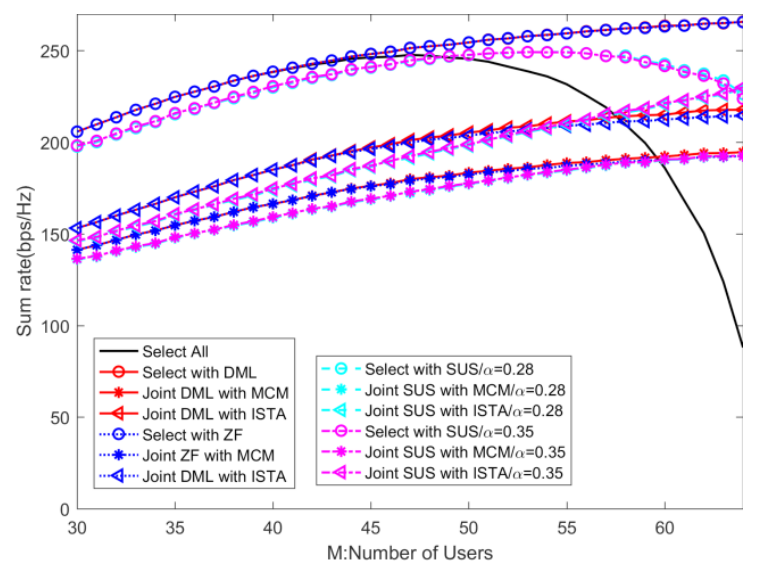

Figure 6. Sum rate performance comparison of different users grouping and antennas selected approach with $N=64, M=30 \sim 64, K=10$ and $S N R=20$.

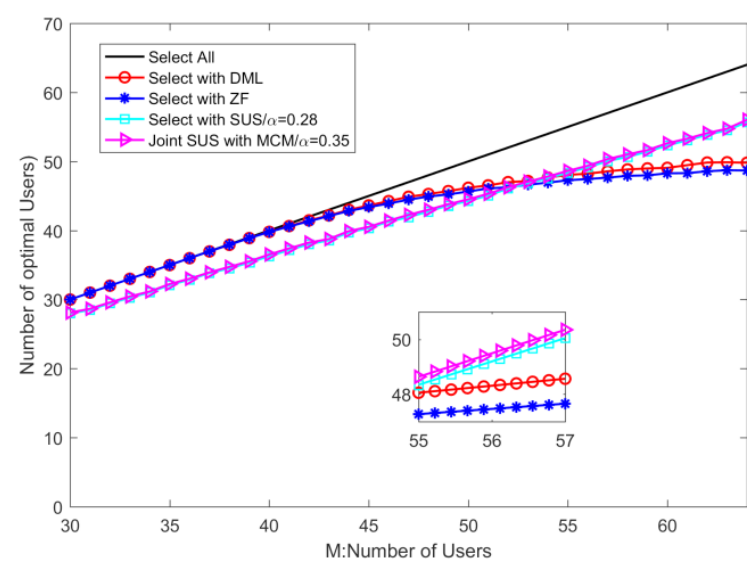

Figure 7. The comparison of the number of optimal user grouping under 'Select All', DML, ZFS, SUS, with $N=64, M=30 \sim 64$ and $S N R=20 d B$.

Figure 8 shows that under different user grouping and antenna selection with $K=30 \sim 50$, selecting $K$ antennas from the massive antennas at BS for each transmit date stream. From it, we can see that the iterative shrinkage thresholding algorithm has a better performance, and greatly reduces the hardware complexity with the $K \mathrm{RF}$ chains. 


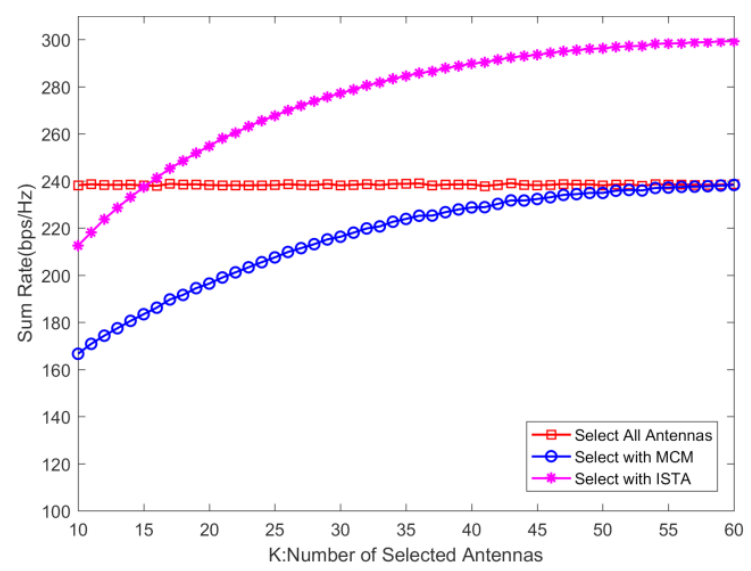

Figure 8. Sum rate performance comparison of different antennas selection: select all antennas, select with MCM, and select with ISTA, with $N=64$, $M=40, K=10 \sim 60$ and $S N R=20 d B$.

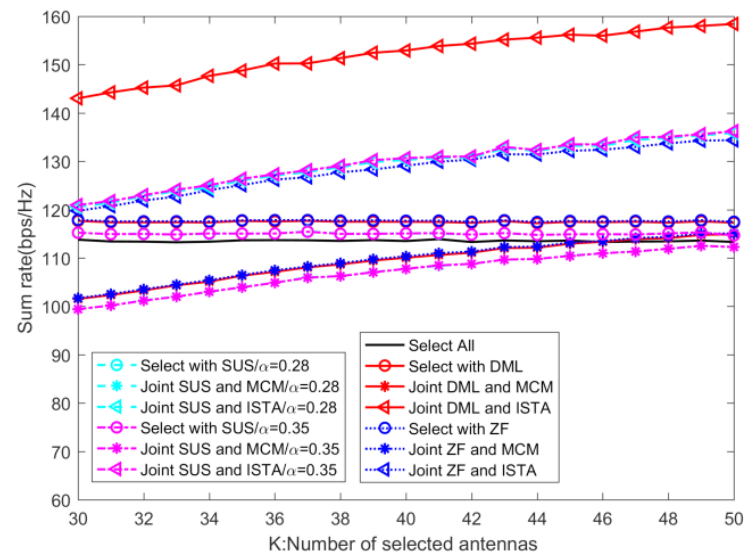

Figure 9. Sum rate performance comparison of different users grouping and antennas selected approach, with $N=64, M=40, K=30 \sim 50$ and

$$
S N R=10 d B \text {. }
$$

\section{Conclusions}

In this paper, a new joint user grouping and antenna selection zero-forcing beamforming algorithm is proposed on the basis of the iterative shrinkagethresholding algorithm. The performance of throughput, computation complexity and hardware complexity are also analyzed. For practically a large number of users and a limited number of RF chains, we delete the minimum effective channel gain to realize the user grouping by employing the concept of orthogonal sub-space orthogonal projection and effective channel gain, and select an optimal antenna subset for each transmit data stream to assign the limited radio-frequency chain dynamically. In this way, the system throughput is maximized with low computation complexity for M-MIMO system. Simulation results verify that the proposed joint beamforming scheme provides a better rate performance with low computational complexity and hardware complexity via a small $K$.

\section{Acknowlegements}

This work is supported by the National Science and Technology Major Project of China (No. 2016ZX03001010-004).

\section{REFERENCES}

1. Beck, A. \& Teboulle, M. (Mar. 2009). A fast iterative shrinkage-thresholding algorithm for linear inverse problems, SIAM Jounal on Imaging Sciences, 2, 183-202.

2. Bjornson, E., Kountouris, M. \& Debbah, M. (2013). Massive MIMO andsmall cells: Improving energy efficiency by optimal softcell coordination. In Proc. $20^{\text {th }} I C T$ (pp.1-5).

3. Guthy, C., Utschick, W. \& Honig, M. L. (2013). Large system analysis of sumcapacity in the gaussian MIMO broadcast channel, IEEE Journal on Selected Areas in Communications, 31(31), 149-159.

4. Habib, A., Krasniqi, B. \& Rupp, M. (2011). Antenna selection in polarization diverse MIMO transmissions with convex optimization. In IEEE Symposium on Communications and Vehicular Technology in the Benelux, SCVT2011 (pp. 663-671). Ghent, Belgium. IEEE.

5. Huang, S., Yin, H., Wu, J. \& Leung, V. C. M. User selection for multiuser MIMO downlink with zero-forcing beamforming, IEEE Trans. Veh. Technol.

6. Huh, H., Caire, G., Papadopoulos, H. C. et al. (2011). Achieving massiveMIMO spectral efficiency with a not-so-large number of antennas, IEEE Transactions on Wireless Communications, 11(9), 3226-3239.

7. Larsson, E. G., Tufvesson, F., Edfors, O. \& Marzetta,T. L. (2014). Massive MIMO for Next Generation Wireless Systems, IEEE Communications Magazine, 52(2), 186-195.

8. Lee, B. M., Choi, J. H., Bang, J. H. et al. (2013). An energy efficient antennaselection for large scale green MIMO systems. In IEEE International Symposium on Circuits and Systems (pp. 950-953). IEEE.

9. Liu, A. \& Vincent, K. N. (2012). Joint power and antenna selection optimizationfor energy-efficient large distributed MIMO networks. In IEEE International Conference 
on Communication Systems, ICCS 2012 (pp. 230-234). Singapore, Singapore. IEEE.

10. Lu, L., Li, G. Y., Swindlehurst, A. L. et al. (2014). An overview of massive MIMO: Benefits and challenges, IEEE Journal of Selected Topics in Signal Processing, 8(5), 742-758.

11. Marzetta, T. L. (2015). Massive MIMO: An introduction, Bell Labs Technical Journal, 20, 11-22.

12. Ngo, H. Q., Larsson, E. G. \& Marzetta, T. L. (2011). Energy and spectral efficiency of very large multiuser MIMO systems, IEEE Transactions on Communications, 61(4), 1436-1449.

13. Phan, K. T. \& Tellambura, C. (2007). Receive Antenna Selection Based on Union-Bound Minimization Using Convex Optimization, IEEE Signal Processing Letters, 14(9), 609-612.

14. Qian, M., Wang, Y., Zhou, Y. et al. (2015). A super BS based centralizednetwork architecture for $5 \mathrm{G}$ mobile communication systems, Digital Communications and Networks, 1(2), 152- 159.

15. Rusek, F., Persson, D., Lau, B. K. et al. (2012). Scaling up MIMO: opportunities and challenges with very large arrays, IEEE Signal Processing Magazine, 30(1), 40-60.

16. Sanayei, S. \& Nosratinia, A. (2004). Antenna selection in MIMO systems, IEEE Commun. Mag., 42(10), 68-73.

17. Su, X., Liang, C. C., Choi, D. M. \& Choi, C. (2016). Power allocation schemes for femtotomacro downlink interference reduction for smart devices inambient intelligence, Mobile Information Systems, vol. 2016, Article ID7172515, 10 pages.

18. Sun, F. G., Liu, J., Xu, H. J. et al. (2008). Receive antenna selection using convex optimization for MIMO systems. In International Conference on Communications and NETWORKING in China (pp. 426-430). Chinacom.

19. Wang, H., Tian, Y. \& Vasseur, C. (2015). NonAffine Nonlinear Systems Adaptive Optimal Trajectory Tracking Controller Design and Application[J], Studies in Informatics and Control, 24(1), 5-11.

20. Xu, G. Z., An, L., Wei, J. et al. (2014). Joint user scheduling and antennaselection in distributed massive MIMO systems with limited backhaulcapacity, Wireless Communication Over ZigBee for Automotive Inclination Measurement China Communications, 11(5), 17-30.

21. Yang, L. X., He, S. W., Wang, Y. et al. (2015). Summary of key technologiesfor $5 \mathrm{G}$ wireless communication system, Journal Of Data Acquisition and Processing, 30(3), 469-485.

22. Yoo, T. \& Goldsmith, A. (Mar. 2006). On the optimality of multi-antenna broadcast scheduling using zero-forcing beamforming, IEEE J. Sel. Areas Commun., 24, 528-542.

23. You, L. \& Gao, X. Q. (2014). The key technologies of massive MIMO wirelesscommunication, ZTE Technology Journal, 20(2), 26-28. 